Author accepted version. Final publication as:

Suerich-Gulick, F., Gaskin, S.J., Villeneuve, M. \& Parkinson, E. (2014) Free surface intake vortices: scale effects due

to viscosity and surface tension, Journal of Hydraulic Research. 52(4): 513-522.

\title{
Free surface intake vortices: \\ Scale effects due to surface tension and viscosity
}

\section{FRANK SUERICH-GULICK,}

Department of Civil Engineering and Applied Mechanics, McGill University, Montréal, Canada

SUSAN J. GASKIN (IAHR Member),

Department of Civil Engineering and Applied Mechanics,

McGill University, MacDonald Engineering Bldg, Room 492, Montréal QC Canada H3A 2K6

Email: susan.gaskin@mcgill.ca (Corresponding Author)

MARC VILLENEUVE, Groupe-Conseil Lasalle, Lasalle, Canada

ÉTIENNE PARKINSON, Andritz Hydro, Vevey, Switzerland

\begin{abstract}
Engineers frequently use physical scale models of hydropower intakes to assess and minimize the occurence of harmful free surface vortices. The impact of surface tension, viscosity and turbulence on the scaling behaviour of the vortices is examined here using an analytical free surface vortex model developed from measurements in a laboratory-scale hydropower intake. First, the effect of surface tension on the free surface depression is computed using a finite-difference model over a wide range of depression scales and shapes. The impact and scaling behaviour of surface tension are found to be qualitatively different depending on whether the depression is dimple- or funnel-shaped. The influence of viscosity on scaling predicted by the analytical vortex model contradicts trends recorded by previous authors, which suggests that additional processes such as turbulent diffusion may play a significant role at larger scales. Scale effects due to the interplay of viscosity and turbulence require further investigation, whereas those due to surface tension are fairly easily quantified and predicted.
\end{abstract}

Keywords: free surface vortex, intake, scale effects, surface tension, turbulence, viscosity 
Free surface intake vortices: Scale effects due to surface tension and viscosity

\section{Introduction}

Free surface vortices upstream from hydropower intakes occasionally cause serious problems in plant operation and it is common practice for engineers to use laboratory-scale physical models to assess and optimize proposed intake designs to maximize flow uniformity and steadiness and minimize the occurrence and intensity of vortices. Detailed measurements in a physical model of a simplified intake (see Fig. 1) were used to develop an analytical vortex model based on Burgers's (1948) vortex model that predicts the vortex's characteristic radius $r_{o}$, bulk circulation $\Gamma_{\infty}$ and tip depth $h_{0}$ (maximum depth of the free surface depression produced by the vortex) in terms of the intake velocity $U_{i}$ and relative intake submergence $s / d$ (Suerich-Gulick, 2013). $s$ is the submergence of the intake pipe and $d$ is its inner diameter. This analytical model is used here to examine and try to quantify how scale effects due to surface tension, viscosity and turbulence affect the translation of vortex characteristics from a laboratory-scale model to the full-scale prototype.

It is widely recognized that the dominant parameter influencing vortex intensity is the Froude number (Quick, 1962; Jain, Raju, and Garde, 1978; Anwar, 1983; Chang and Prosser, 1987), defined as $\mathrm{F}_{d}=U_{i} /(g d)^{1 / 2}$ or $\mathrm{F}_{s}=U_{i} /(g s)^{1 / 2}$, where $g$ is the gravitational acceleration. Since the exact dependence on Froude number varies with the intake configuration and geometry (Knauss, 1987), it is common practice to assess and optimize proposed intakes using laboratory-scale models operated at Froude similitude such that $F_{M}=F_{P}$, where $F_{M}$ and $F_{P}$ are the laboratory model and prototype values, respectively (Quick, 1962; Chang and Prosser, 1987). Since water is used in the laboratory model, it is impossible to match the Weber (W), Reynolds (R) and Froude numbers simultaneously, leading to uncertainty about scale effects which has yet to be fully resolved (Tastan and Yıldirım, 2010). The intake definitions for $\mathrm{W}$ and $\mathrm{R}$ are used here, using the submergence $s$ as the characteristic length: $\mathrm{W}_{s}=\rho U_{i}^{2} s / \sigma$ and $\mathrm{R}_{s}=U_{i} s / v$, where $\rho, v$ and $\sigma$ are the water density, kinematic viscosity, and the air-water surface tension coefficients, respectively. Some authors define $\mathrm{W}_{d}$ and $\mathrm{R}_{d}$ using the intake diameter $d$ instead of the intake submergence $s$, indicated by a subscript.

Scale effects due to viscosity and surface tension have often been studied by documenting how the critical condition in physical models varies with increasing Reynolds and Weber numbers. Most authors define the critical condition for air entrainment as the operating condition at which the tip of the free surface depression just reaches the intake pipe (Daggett and Keulegan, 1974; Jain et al., 1978; Anwar, 1983; Odgaard, 1986; Gulliver, 1988; Hite and Mih, 1994; Möller et al., 2012). A common approach is to try to identify a minimum $\mathrm{W}$ above which surface tension effects can be neglected (Daggett and Keulegan, 1974; Jain et al., 1978; Anwar and Amphlett, 1980; Anwar, 1983), or a minimum $R$ above which viscous effects can be neglected (Daggett and Keulegan, 1974; 
Jain et al., 1978; Anwar, 1983; Padmanabhan and Hecker, 1984; Chang and Prosser, 1987; Tastan and Yildirım, 2010).

Independence from surface tension effects in experiments has been reported at $\mathrm{W}_{d}$ values above 120 (Jain et al., 1978), 600 (Padmanabhan and Hecker, 1984), and 748 (Möller et al., 2012), while Anwar (1983) reported persisting surface tension effects for $\mathrm{W}_{s}$ values up to $1.5 \times 10^{4}$ for dimple depressions and up to $4 \times 10^{4}$ for air core vortices, which have deep narrow funnels that reach far below the free surface. Tastan and Yıldırım (2010) observed that limiting values for $R$ and $\mathrm{W}$ depend on both flow and geometrical conditions in experiments. Using theoretical analysis, Odgaard (1986) concluded that surface tension effects should be negligible for $\mathrm{W}_{d}>720$. Other authors have examined surface tension effects by numerically computing the profile of the free surface depression by the finite difference method or analytical or series approximations (Y1ldirım and Jain, 1981; Andersen et al., 2006; Stepanyants and Yeoh, 2008a; Ito et al., 2010).

Reynolds number dependence has been observed to decrease asymptotically with increasing R (Daggett and Keulegan, 1974; Jain et al., 1978; Anwar, 1983; Chang and Prosser, 1987), suggesting viscous effects may be negligible beyond a threshold $R$ value. Suggested minimum values for $\mathrm{R}$ range from $4 \times 10^{4}$ to $1.4 \times 10^{5}$, depending on the geometry and the Froude number, using various definitions of R (Daggett and Keulegan, 1974; Jain et al., 1978; Anwar, 1983; Padmanabhan and Hecker, 1984; Chang and Prosser, 1987; Tastan and Yıldırım, 2010). Detailed data needed to explain this trend are lacking.

Greater understanding of the processes driving scale effects should help engineers to interpret vortex observations more appropriately or estimate correction factors when the laboratory-scale model does not meet recommended $\mathrm{W}$ and $\mathrm{R}$ values. This scenario occurs for particularly large prototype intakes and/or when the model must include a significant stretch of the upstream river reach to capture approach flow conditions.

Odgaard (1986) proposed that the asymptotic $R$ trend is due to turbulent mixing that enhances the effective diffusivity in the vortex core. He models the intake vortex using Burgers's (1948) vortex model in combination with a simple eddy diffusivity model. Burgers's model assumes that the radial profiles of the azimuthal $V_{\theta}(r)$ and radial $V_{r}(r)$ velocities in the vortex are constant along the vortex axis $z$ and that the axial velocity $V_{z}(z)$ is independent of $r$ and varies linearly with $z$ :

$$
\begin{array}{rlrl}
V_{\theta}(r) & =\frac{\Gamma_{\infty}}{2 \pi r}\left[1-\exp \left\{-\left(r / r_{o}\right)^{2}\right\}\right], & & \\
V_{z}(z) & =a z, & V_{r}(r)=-a r / 2, \\
r_{o} & =2(v / a)^{1 / 2}, & a & =\partial V_{z} / \partial z,
\end{array}
$$

where $r, \theta, z$ are the radial, azimuthal and axial cylindrical coordinates with $z$ aligned with the vortex axis pointing down from the free surface, and $V_{r}, V_{\theta}, V_{z}$ are the corresponding velocities. The 
gradient $a$ is a constant with units $\mathrm{s}^{-1}, \Gamma_{\infty}$ (units $\mathrm{m}^{2} \mathrm{~s}^{-1}$ ) is the bulk circulation of the vortex, and $v$ (units $\mathrm{m}^{2} \mathrm{~s}^{-1}$ ) is the kinematic viscosity of the fluid. Burgers's model is based on the hypothesis that a stable vortex with a constant vorticity and $V_{\theta}(r)$ profile along the vortex axis is produced by an equilibrium of axial stretching $\partial V_{z} / \partial z$ and radial viscous diffusion. Detailed measurements of the velocity field of a free surface intake vortex suggest that the model captures the basic vortex structure quite well (Suerich-Gulick, 2013) even if some subtle discrepancies exist.

Odgaard (1986) suggests that momentum mixing in the vortex caused by turbulence increases the effective viscosity $v_{\text {eff }}$ at larger scales. He replaces $v$ in Burgers's expression for $r_{o}$ (equation 3) by $v_{\mathrm{eff}}=v+v_{\mathrm{T}}$, and assumes that the eddy diffusivity $v_{\mathrm{T}}$ scales as $v_{\mathrm{T}}=\chi \Gamma_{\infty}$, following Squire (1965), with the non-dimensional constant $\chi=6 \times 10^{-5}$. The resulting model predicts Jain et al.'s (1978) critical submergence measurements fairly well (Gulliver, 1988). Hite and Mih (1994) follows the same approach.

The proposal that $v_{\text {eff }}$ increases with $\Gamma_{\infty}$ would appear to contradict past results that radial turbulent fluctuations are suppressed by flow rotation (Bradshaw, 1973; Spalart, 1998; Jacquin and Pantano, 2002; Suerich-Gulick, 2013) and that the spreading rate in the case of wing tip vortices is governed by viscous diffusion rather than by turbulent mixing (Zeman, 1995). However it is possible that that radial turbulent diffusion is suppressed to a lesser degree at larger scales, as both $\Gamma_{\infty} / v$ and $\mathrm{R}$ increase. Increasing eddy diffusivity would produce a gradual decrease in the relative contribution of molecular viscosity $v$ to the effective diffusivity, until 'viscous effects' become negligible at $\mathrm{R}_{d}$ values on the order of $10^{5}$ (Odgaard, 1986). Although our measurements indicate that that the contribution of turbulent mixing to radial diffusion of the vortices is negligible under the operating conditions examined (Suerich-Gulick, 2013), the maximum $v_{\mathrm{T}}$ values from Odgaard (1986)'s model would only reach half the molecular viscosity, producing a $22 \%$ increase in the core radius $r_{o}$ (equation 3). This is comparable to the variation in $r_{o}$ observed experimentally in Suerich-Gulick (2013).

\section{Method}

\subsection{Experiment}

A laboratory-scale model of a simplified low-head hydropower intake is constructed with two tall pier-like plates mounted perpendicular to the downstream wall of the channel, one on each side of the intake opening, as shown in Fig. 1. Each pier produces two vortices in its wake: a submerged vortex with one end connected to the channel bed, and a free surface vortex with one end connected to the free surface. The other end of each vortex is drawn into the intake pipe. We study a range of vortex intensities, ranging from an imperceptible dimple to a funnel vortex that regularly entrains air bubbles. The details of the experimental setup and the eight operating conditions studied are described in Suerich-Gulick (2013). 

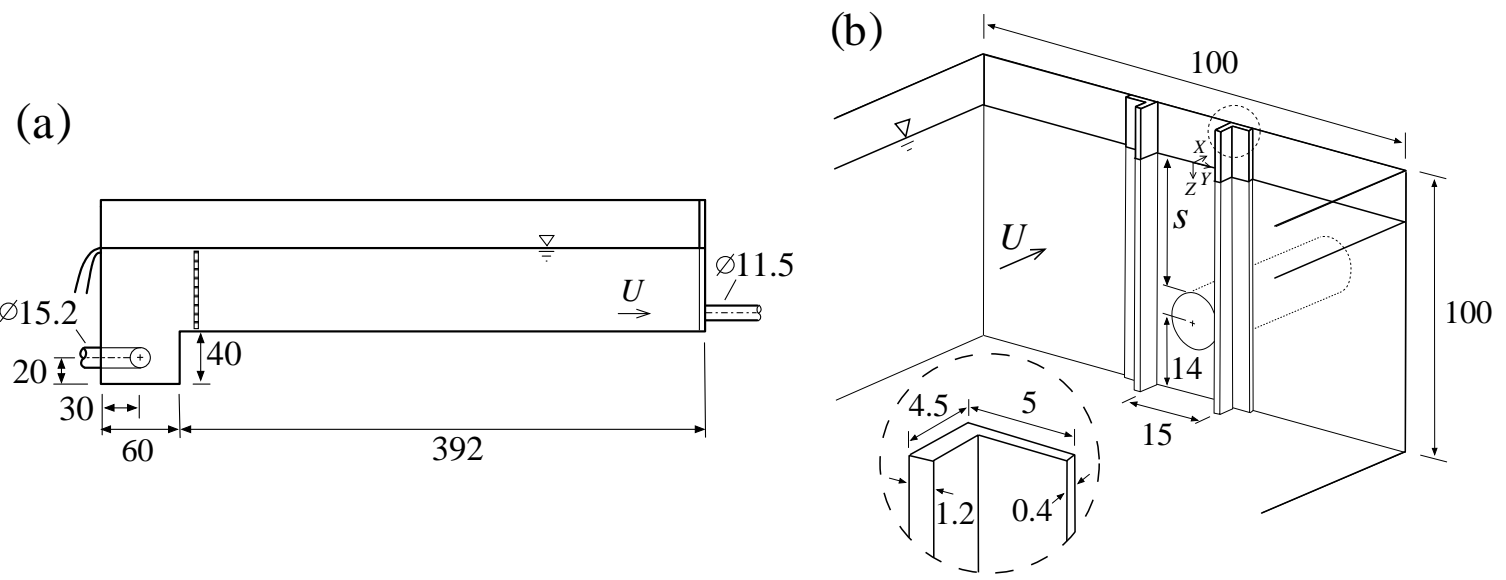

Figure 1 (a) Vertical section and (b) isometric views of the laboratory model (dimensions in $\mathrm{cm}$ ), adapted from Suerich-Gulick et al. (2014).

A high-speed video camera is used to simultaneously record the particle trajectories of the free surface vortex produced by the right pier (defined looking downstream) and the profile of the free surface depression it produces. The profiles of $V_{\theta}(r)$ and $V_{z}(z)$ are then computed using particle tracking velocimetry (PTV) and Burgers's profile (equation 1) is fitted to the measured profiles. The results are presented and discussed in Suerich-Gulick (2013). In this paper, we numerically compute the free surface depression that should be produced by the measured velocity profile $V_{\theta}(r)$ including surface tension, and we compare it with the actual free surface depression recorded at the same time as the velocity. Once we have ascertained in this manner that the numerical model predicts the free surface depression with sufficient accuracy, we do a parametric study using the numerical model to gain insight into the relative impact of surface tension for different shapes and scales of the free surface depression.

\subsection{Free surface profile computations}

At small scales, surface tension can significantly reduce the depth of the free surface depression produced by the vortex. This effect is highly nonlinear since it both modifies and is controlled by the local curvature of the free surface. We examine the scaling behaviour of surface tension by numerically computing the depression profile $h(r)$ with $\left(h_{\sigma}\right)$ and without $\left(h_{\mathrm{n}}\right)$ surface tension over a wide range of vortex intensities produced by different combinations of $\Gamma_{\infty}$ and $r_{o}$. The evolution of the relative difference $\delta \equiv \Delta h / h_{\mathrm{n}, 0}$ between the profile tip depths is examined, where $\Delta h \equiv h_{\mathrm{n}, 0}-h_{\sigma, 0}$ (Yıldırım and Jain, 1981).

The free surface profile is controlled by the equilibrium of the forces exerted by gravity, centripetal acceleration and surface tension. Following Andersen et al. (2006), Stepanyants and Yeoh (2008a) and Ito et al. (2010), we use Laplace's model (Laplace, 1807) that surface tension 
reduces the pressure across the air-water interface by $l_{\sigma}^{2} \kappa(r)$, where $\kappa(r)$ is the local mean curvature of the air-water interface and $l_{\sigma}^{2}=\sigma /(\rho g)$ is the squared characteristic length of the air-water interface. The resulting radial profile of the depression $h_{\sigma}(r)$ is given by

$$
h_{\sigma}(r)=\int_{\infty}^{r}\left(\frac{V_{\theta}(\dot{r})^{2}}{g \dot{r}}-l_{\sigma}^{2} \kappa(\dot{r})\right) \mathrm{d} \dot{r}
$$

(Andersen et al., 2006). A constant value for $l_{\sigma}$ of $2.73 \mathrm{~mm}$ is used here, which corresponds to a clean air-water interface at $15^{\circ} \mathrm{C}$. The variations in $l_{\sigma}$ associated with the range of experimental temperatures $\left(13\right.$ to $15^{\circ} \mathrm{C}$ ) are negligible compared to those that might be caused by impurities in the water or floating on the free surface. The kinematic energy associated with $V_{z}$ and $V_{r}$ would slightly increase the depth of the depression, but this contribution is negligible compared to that of $V_{\theta}$ (Odgaard, 1986). We assume that the curved path of the vortex axis entering the intake pipe has a negligible impact on the free surface depression, but it might be more signficant for shallower submergence values or a different geometry.

The mean local curvature $\kappa(r)$ is given by

$$
\kappa(r)=-\frac{1}{2}\left\{\frac{h_{r}}{r\left[1+\left(h_{r}\right)^{2}\right]^{1 / 2}}+\frac{h_{r r}}{\left[1+\left(h_{r}\right)^{2}\right]^{3 / 2}}\right\},
$$

where $h_{r}$ and $h_{r r}$ are the first and second derivatives of $h$ with respect to $r$ respectively (Andersen et al., 2006). The first term on the right is the curvature about the horizontal axis (perpendicular to the page in a 2D section of the profile such as Fig. $4 \mathrm{a}$ and the second term is the curvature about the vortex's (vertical) axis of rotation. The free surface profile has a positive (concave) horizontal axis curvature at the vortex tip, then some distance beyond $r>r_{o}$ it passes through an inflection point and the horizontal axis curvature becomes negative (convex). The surface tension force thus pushes the interface upward in the core portion of the vortex and pulls it down very slightly just outside the core.

Since our primary goal is to get a larger view of trends in surface tension effects over a range of shapes and scales rather than to obtain the exact shape of the depression, the free surface profile is computed by directly substituting Burgers's relation for $V_{\theta}(r)$ from equation (1) into equation (4):

$$
h_{\sigma}(r)=\int_{\infty}^{r}\left\{\frac{\Gamma_{\infty}^{2}}{4 \pi^{2} g \dot{r}^{3}}\left[1-\exp \left(-\left(\dot{r} / r_{o}\right)^{2}\right)\right]^{2}-l_{\sigma}^{2} \kappa(\dot{r})\right\} \mathrm{d} \dot{r},
$$

This approximation neglects the effect of the free surface depression on the velocity field and hence indirectly on the depression itself as well. Stepanyants and Yeoh's (2008b) results suggest that this approximation produces a negligible error in $\delta$ in the case of a mild dimple depression and an error of $26 \%$ for an extremely deep, funnel-type depression with a nominal free surface depression slope $\zeta \equiv h_{\mathrm{n}, 0} / r_{o}=110$. This is judged to be an acceptable level of error for the purpose of this study.

Equation (6) is discretized along $r$ by central differences and an equilibrium profile $h_{\sigma}(r)$ is computed numerically for the given $r_{o}$ and $\Gamma_{\infty}$ by gradually decreasing $r_{o}$ from a large value (which 
produces a very shallow depression) to the desired $r_{o}$. Funnel vortices are characterized by a large peak in surface curvature at the tip $(r=0)$, requiring a large relaxation factor and smoothing of the computed curvature profile $h_{\sigma}(r)$ between each iteration to suppress oscillations there.

\section{Analysis and discussion}

\subsection{Surface tension effects}

The free surface profile code is first tested using the free surface and velocity profiles measured and computed by Andersen et al. (2006) for a moderate funnel of nominal depression slope $\zeta=15$ and the results compare very well. The code is then tested by computing the free surface depression from the measured velocity profiles in our experiment, where surface tension effects are significant, and comparing the computed tip depth $h_{0 \text {,comp }}$ to that recorded in the film segments.

Figure 2 a shows each measured tip depth $h_{0 \text {,exp }}$ compared to the tip depth $h_{0, \text { comp }}$ computed using equation (6) with $\Gamma_{\infty}$ and $r_{o}$ obtained by fitting Burgers's profile (equation 1) to the measured $V_{\theta}(r)$ profiles. The shape of the datapoints indicates the intake velocity $U_{i}$ and the shade indicates the relative submergence $s / d$. The horizontal error bars show the spread between the two values of $h_{\sigma, 0}$ computed from $r_{o}$ and $\Gamma_{\infty}$ obtained from the two fitting methods, and the symbols show the mean. Although the spread is somewhat large for some points, the agreement is close enough to indicate that both the method used to measure the azimuthal velocities and Burgers's model used to describe the measured profiles are suffiently accurate to predict the free surface depression from the velocity measurements.

The code is then used to compute the free surface depression with and without surface tension produced by Burgers's vortices with a range of $\Gamma_{\infty}$ and $r_{o}$ values. As shown in Fig. $2 \mathrm{~b}$, the results reveal that the relative surface tension effect $\delta$ scales very differently depending on the shape of the depression, which is quantified here using the nominal depression slope $\zeta$. The transition between the dimple and funnel modes occurs around $1<\zeta<10$, depending on the scale. For dimple-shaped depressions corresponding to $\zeta \lesssim 1-10, \delta$ becomes independent of $\zeta$, while for funnel-shaped depressions ( $\zeta \gtrsim 1-10$ ), $\delta$ varies with both the scale and shape $\zeta$. Furthermore, the data show that for a given scale $r_{o} / l_{\sigma}$, the relative surface tension effect $\delta$ is much more significant in a dimple than in a funnel vortex, as obtained by Yıldırım and Jain (1981).

Figure 3a shows that once the limiting dimple shape is reached (towards the upper right of the graph), $\delta$ converges to a unique function $f_{\sigma}$ for the different $\Gamma_{\infty}$ values that depends only on the scale $r_{o} / l_{\sigma}$ :

$$
f_{\sigma}\left(r_{o} / l_{\sigma}\right)=\left[\exp \left(-0.44\left(r_{o} / l_{\sigma}\right)^{2}\right)+1.9\left(r_{o} / l_{\sigma}\right)^{1.6}\right]^{-1},
$$

which at large scales $\left(r_{o} / l_{\sigma} \gtrsim 3\right)$ tends towards a straight line $\delta \sim\left(r_{o} / l_{\sigma}\right)^{2(-0.9)} \sim\left(r_{o} / l_{\sigma}\right)^{-1.8}$. Conversely, when $\delta$ is plotted in Fig. $3 \mathrm{~b}$ as a function of the product $\left(r_{o} / l_{\sigma}\right)^{2} \zeta=r_{o} h_{\mathrm{n}, 0} / l_{\sigma}^{2}$, the curves collapse at large values (corresponding to the funnel shape) to a straight line of slope $\delta \sim\left(r_{o} h_{\mathrm{n}, 0} / l_{\sigma}^{2}\right)^{-0.6}$. 
(a)

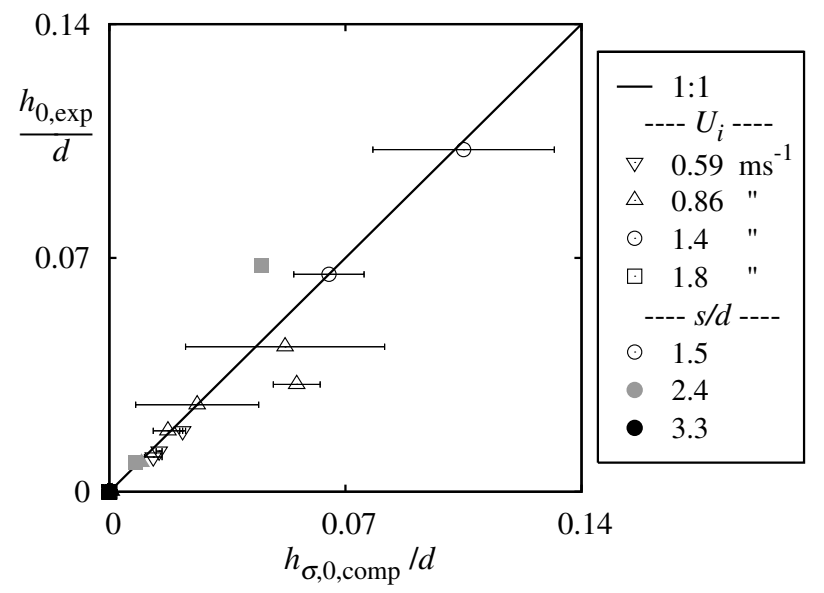

(b)

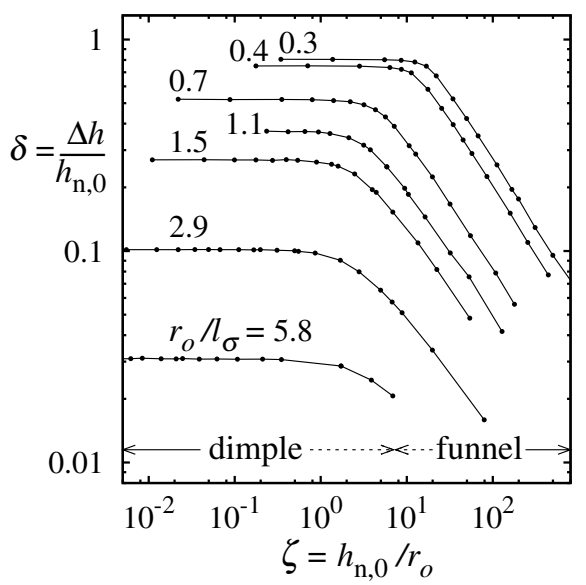

Figure 2 (a) Correspondence between the measured and computed tip depths. (b) Relative surface tension effect as a function of the depression scale and nominal slope, adapted from Suerich-Gulick et al. (2014).

The dashed lines in Fig. 3a-b indicate lines of constant bulk circulation $\Gamma_{\infty}$, while the solid lines in Fig. $3 b$ indicate lines of constant scale $r_{o} / l_{\sigma}$.

These trends can be compared to the scaling behaviour of the local curvature $\kappa_{\mathrm{n}, 0}$ of the free surface at $r=0$ in the absence of surface tension (equation 5) in the limits $\zeta \ll 1$ (dimple) and $\zeta \gg 1$ (funnel). If $r$ is assumed to scale with $r_{o}, h_{r}$ with $\zeta$ and $h_{r r}$ with $h_{\mathrm{n}, 0} / r_{o}^{2}$, then equation (5) produces $\kappa_{\mathrm{n}, 0} \sim h_{\mathrm{n}, 0} / r_{o}^{2}$ for the dimple and $\kappa_{\mathrm{n}, 0} \sim r_{o}^{-1}$ for the funnel. If it is further estimated that $\delta \sim \kappa_{\mathrm{n}, 0} l_{\sigma}^{2} / h_{\mathrm{n}, 0}$, this produces the scaling behaviour $\delta \sim\left(l_{\sigma} / r_{o}\right)^{2}$ for the dimple and $\delta \sim l_{\sigma}^{2} /\left(r_{o} h_{\mathrm{n}, 0}\right)$ for the funnel (the relation used by Odgaard (1986) in his analysis). So the same essential scaling behaviour is produced by the computations and the theoretical analysis, except that the slopes of the computed trends ( -1.8 and -0.6 for the dimple and funnel respectively) are weaker than those ( -2 and $-1)$ produced by the rough theoretical analysis. Stepanyants and Yeoh (2008a) also obtain $\delta \sim r_{o}^{-2}$ for the dimple. The difference between our numerical result and that of Stepanyants and Yeoh (2008b) might be due to simplifications in the velocity profile model used here, or to the different solution methods, since Stepanyants and Yeoh use a series solution with analytical functions to approximate the shape of the tip instead of computing a discretized profile. However it seems quite possible that the difference in slopes between our computed trend and the present theoretical analysis is due to physics rather than numerical error, since the theoretical analysis does not capture the non-linearity of the process by which surface tension changes the shape of the free surface depression, which in turn affects the magnitude of the surface tension and so forth.

To understand these trends, we examine how the computed shape of the free surface depression is modified by surface tension and how that effect depends on the initial shape and scale 
(a)

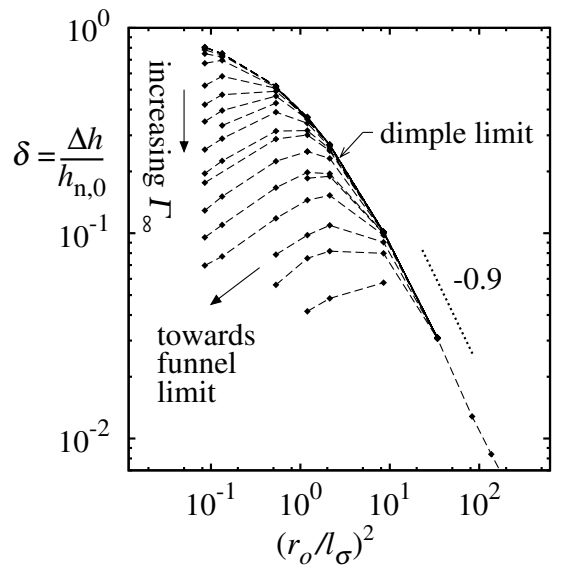

(b)

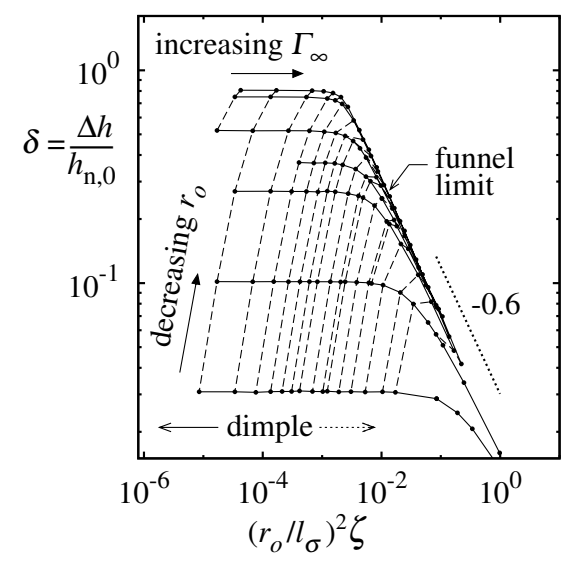

Figure 3 The influence of (a) scale and (b) the combined scale and shape on the relative surface tension effect.

of the depression. Comparison of the profiles in Fig. 4a computed with surface tension (dashed and dotted lines) and without (solid line) shows that surface tension acts differently on different regions of the profile depending on the shape of the depression. Within a given scale $\left(r_{o} / l_{\sigma}=0.3\right)$ there is a much stronger relative reduction of the depression $h_{\sigma} / h_{\mathrm{n}, 0}$ for the dimple vortices $(\zeta \lesssim 20)$ than for the funnel vortices $(\zeta \gtrsim 20)$. Surface tension acts strongly over a much larger radius in the dimple vortex (up to $r / r_{o} \approx 2$ ) than in the funnel vortex where the effect is restricted to an inner region $r / r_{o} \lesssim 0.5$ that shrinks as the funnel gets deeper. In the funnel vortices, surface tension appears to essentially clip off the tip of the depression, thereby significantly diminishing the spike in curvature at the tip that characterizes funnel vortices. This can be seen in Fig. 4b, which shows the curvature profiles $\kappa_{\sigma}\left(r / r_{o}\right)$ that correspond to the free surface profiles in Fig. 4a. In the absence of surface tension (solid line), $r_{o} \kappa$ for $\zeta=350$ reaches a peak of 510 at $r=0$. Figure $4 \mathrm{c}$ shows that surface tension has much less effect at a larger scale $\left(r_{o} / l_{\sigma}=1.5\right)$ in both the dimple ( $\zeta \lesssim 5$ here) and funnel vortices. The profiles for the two lowest values of $\zeta$ in Figs. $4 \mathrm{a}$ and Figs. $4 \mathrm{c}$ coincide, indicating that the dimple shape limit has been reached, where $\delta$ becomes independent of $\zeta$.

These results clearly demonstrate that surface tension effects do not scale in the same way for dimple vortices and funnel vortices. This qualitative difference in behaviour might partly explain the variability in recommendations found in the literature for the minimum laboratory model size required to avoid surface tension effects. The results also strongly suggest that empirical surface tension scaling laws derived by studying the onset of air entrainment (ie. deep funnel vortices) must not be directly employed to interpret vortex observations in reduced scale models of hydropower plants, where spatial constraints are such that only dimple vortices are commonly observed. 
(a)

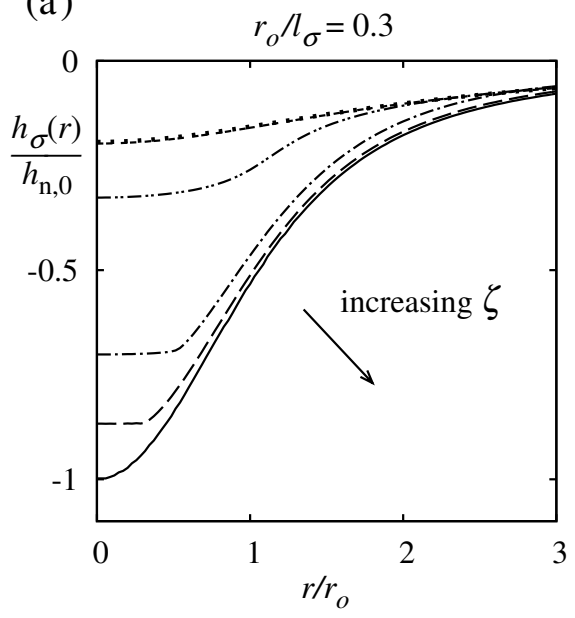

(c)

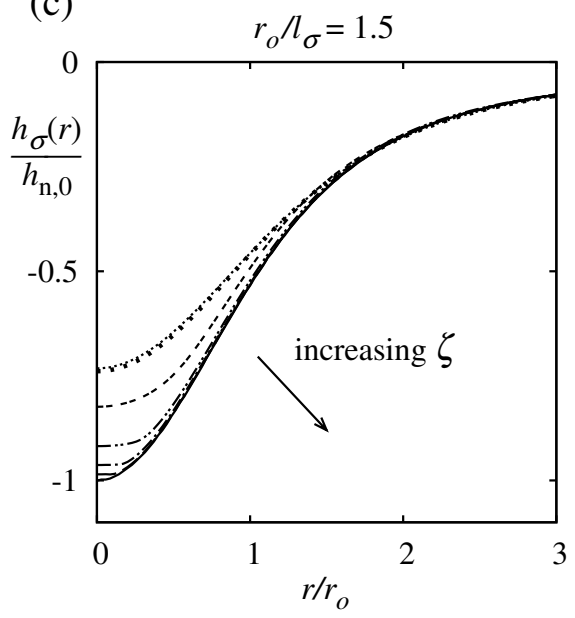

(b)

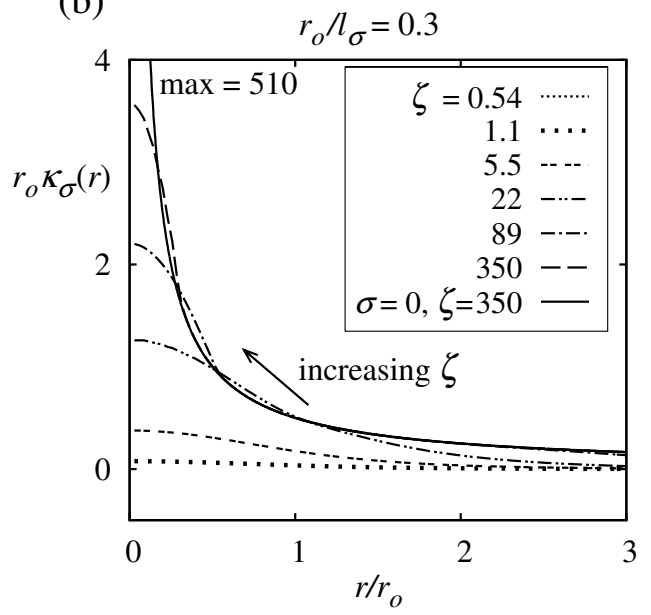

Figure 4 Effect of surface tension on $(a, c)$ the profile of the free surface depression and (b) the curvature of the free surface for different depression shapes and scales. 


\subsection{Scale effects associated with viscosity and turbulence}

In equation(4.9) of Suerich-Gulick (2013), the range of expected tip depths $h_{\mathrm{n}, 0, \text { est }}$ for a specific geometry was estimated as a function of the intake velocity $U_{i}$ and relative submergence $s / d$. We obtain $h_{\sigma, 0, \text { est }}$ including surface tension by adding the surface tension correction function $f_{\sigma}$ (equation 7):

$$
\frac{h_{\sigma, 0, \text { est }}}{d}=\frac{\mathrm{c}_{0}\left(1-f_{\sigma}\right)}{(1-\beta)} \mathrm{R}_{s} \mathrm{~F}_{s}^{2}\left(\frac{d}{k}\right)^{3}\left(\frac{l_{p}}{s}\right)^{2}\left(\frac{d}{\mathrm{c}_{4} s}-\mathrm{c}_{2}\right)^{2},
$$

with the non-dimensional coefficients $\mathrm{c}_{0}=3.7 \times 10^{-5}$ and $\mathrm{c}_{2}=0.28 . \mathrm{c}_{4}$ has two values, 1.0 and 0.6 , corresponding to low and high values of $\Gamma_{\infty}$, respectively. $l_{p}$ is the length of each pier, $k$ is the distance between the piers, and $\mathrm{R}_{s} \mathrm{~F}_{s}^{2}=\mathrm{R}_{d} \mathrm{~F}_{d}^{2}=U_{i}^{3} /(v g)$. This expression is valid for all the conditions examined, regardless of vortex shape, except that $f_{\sigma}$ (equation 7 ) is valid only for dimple-type depressions and will overestimate the surface tension effect for funnel- and transitiontype depressions. For a more accurate correction for these latter shapes, $\delta$ can be read off Fig. $2 \mathrm{~b}$ for a given nominal depression slope $\zeta$ and scale $r_{o} / l_{\sigma}$ and substituted for $f_{\sigma}$ in equation (8). The solid lines indicate curves of constant $r_{o} / l_{\sigma}$.

Figure 5a shows the measured values compared with the range of values $h_{\sigma, 0, \text { est }}$ estimated from equation (8) with $r_{o}$ in $f_{\sigma}$ (equation 7) estimated using $\beta=0$. Surface tension has a minimal impact relative to the variability associated with variations in $r_{o}$ and $\Gamma_{\infty}$. The predicted range of $h_{\sigma, 0, \text { est }}$ values is shown by the boxes: the upper limit is obtained with $\mathrm{c}_{4}=0.6$ and $\beta=0.15$ and the lower limit is obtained with $\mathrm{c}_{4}=1.0$ and $\beta=0.85$. Possible causes of $\Gamma_{\infty}$ variability include as-yet poorly understood strengthening processes within the vortex, and turbulent fluctuations in the surrounding flow. Variability in $r_{o}$ would appear to be mainly due here to variations in the shape of the $V_{z}(z)$ profile in the upper portion of the flow, ranging between a more linear $\left(V_{z}(z) \sim z\right)$ and a more non-linear one $\left(V_{z}(z) \sim(h-z)^{-1}\right)$. This effect is indicated in equation (8) by the parameter $\beta$. As discussed in Suerich-Gulick (2013), $\beta$ indicates the extent of the linear profile of $V_{z}(z)$ : where $0 \leq \beta \lesssim(1-0.5 d / s)$ is a proportion of the distance $s$ from the free surface to the top of the inlet. $\beta=0$ indicates that $V_{z}(z)$ inside the vortex follows the non-linear profile of the flow outside the vortex, while larger values of $\beta$ indicate that $V_{z}(z)$ follows a linear profile in $z$ from the free surface over a greater distance, producing a steeper slope in $V_{z}(z)$ at the free surface. In the experiment, $\beta$ is observed to fall in the range $0.15 \leq \beta \leq 0.85$ (Suerich-Gulick, 2013). Turbulence in the vortex core might also produce variations in $r_{o}$ by enhancing the effective radial diffusivity in equation (3).

To compare equation (8) with other results, it is reformulated in terms of the critical relative submergence $s_{c}^{\prime}$ for air entrainment, where the tip of the vortex depression reaches the top of the outlet so that $\left.h_{\sigma, 0}\right|_{\text {crit }}=s$. Substituting $\left.s_{\mathrm{c}}^{\prime} \equiv(s / d)\right|_{\text {crit }}$ for $h_{\sigma, 0} / d$ in equation (8) and isolating terms in $s_{\mathrm{c}}^{\prime}$ produces 


$$
\frac{s_{\mathrm{c}}^{\prime 5}}{\left(\mathrm{c}_{4}^{-1}-\mathrm{c}_{2} s_{\mathrm{c}}^{\prime}\right)^{2}}=\frac{\mathrm{c}_{0}\left(1-f_{\sigma}\right)}{(1-\beta)}\left(\frac{d l_{p}^{2}}{k^{3}}\right) \mathrm{R}_{d} \mathrm{~F}_{d}^{2} .
$$

As shown in Fig. 5b, this relation predicts that $s_{\mathrm{c}}^{\prime}$ scales roughly as $s_{\mathrm{c}}^{\prime} \sim A \mathrm{R}_{d}^{1 / 6} \mathrm{~F}_{d}^{1 / 3}$ at smaller values of $\mathrm{R}_{d}^{1 / 2} \mathrm{~F}_{d}$, with $A^{2}=\mathrm{c}_{0}\left(1-f_{\sigma}\right)(1-\beta)^{-1}\left(d l_{p}^{2} / k^{3}\right)$. It then flattens out at larger values, becoming less sensitive to $\mathrm{R}_{d}^{1 / 2} \mathrm{~F}_{d}$ at deeper submergences. The two curves are produced by the two values of $\mathrm{c}_{4}(0.6$ and 1$)$, and they have a singularity at $s_{\mathrm{c}}^{\prime}=6.0$ and 1.7 , respectively. The shift to a milder slope at larger $F_{d}$ reproduces trends observed in physical models of vertical intakes by Tastan and Yıldırım (2010) and of horizontal intakes by Jiming et al. (2000). It differs from the trends with a constant slope on a log-log scale found by other researchers. Gulliver (1988) observed $s_{c}^{\prime} \sim \mathrm{F}_{d}^{2 / 3}$ in experiments with vertical intakes, and Rao et al. (1997) derived the same relation from Yildırım and Kocabaş's (1995) analysis of lateral intakes in crossflow or at the end of a channel. Jain et al. (1978) observed $s_{c}^{\prime} \sim \mathrm{F}_{d}^{0.5} N_{\Gamma *}^{0.42} K^{-1}$ and Odgaard (1986) analytically derived $s_{\mathrm{c}}^{\prime} \sim N_{\Gamma *}^{1 / 2} \mathrm{R}_{d}^{1 / 4} \mathrm{~F}_{d}^{1 / 2}$, both for flow in a cylinder, where $N_{\Gamma *}=\Gamma_{\infty} S / Q$ is the non-dimensional circulation and $K$ is a viscous correction factor.

The decreased sensitivity to $F_{d}$ predicted by equation (9) at greater $F_{d}$ values is due to the non-linear $V_{z}(z)$ profile that roughly follows $V_{z} / U_{i} \sim d /(s-z)$, as opposed to the linear profile $V_{z} / U_{i}=z / s$ assumed by Odgaard (1986). The non-linear profile has a much milder gradient $\partial V_{z} / \partial z$ at the free surface at large submergences, which produces more diffuse and thus weaker vortices, requiring a greater relative increase in $F_{d}$ to produce an air core than at lower submergences. The linear profile assumed by Odgaard (1986) in his model might explain why it tends to overpredict $s_{\mathrm{c}}^{\prime}$ compared with observations (Jain et al., 1978) at larger $s_{\mathrm{c}}^{\prime}$ values in several configurations (Gulliver, 1988). It should also be noted that $\Gamma_{\infty}$ is dependent on $U_{i}, s / d$ and the relative pier length $l_{p} / d$ in the current experiment, whereas it is imposed using adjustable guide vanes in the experiments considered by Odgaard (1986) and Jain et al. (1978), which should affect the corresponding scaling relations.

Equation (8) can also be used to evaluate how the characteristics of a free surface vortex in a laboratory-scale model operated at Froude similitude would translate to a much larger prototype intake with the same geometry with a geometric scaling ratio $\alpha=\ell_{\mathrm{P}} / \ell_{\mathrm{M}}$, where $\ell_{\mathrm{M}}$ and $\ell_{\mathrm{P}}$ are the characteristic lengths in the laboratory model and the prototype respectively. Neglecting surface tension $\left(1-f_{\sigma}\right)$ and assuming the model and prototype are geometrically identical so that $(d / k)$, $(d / s)$, and $\left(l_{p} / k\right)$ are identical in both the model and prototype, equation (8) becomes

$$
h^{\prime} \equiv \frac{h_{\mathrm{n}, 0}}{d} \sim \frac{\mathrm{c}_{0}}{(1-\beta)}\left(\frac{d}{\mathrm{c}_{4} s}-\mathrm{c}_{2}\right)^{2} \mathrm{~F}_{s}^{2} \mathrm{R}_{s} .
$$

Since $\mathrm{F}_{\mathrm{M}}=\mathrm{F}_{\mathrm{P}}$, the ratio of the outflow velocities $U_{i, \mathrm{P}} / U_{i, \mathrm{M}}=\alpha^{1 / 2}$, and because water is usually employed in the laboratory model, $v_{\mathrm{M}}=v_{\mathrm{P}}$ and the prototype-to-model ratio of the Reynolds num- 
(a)

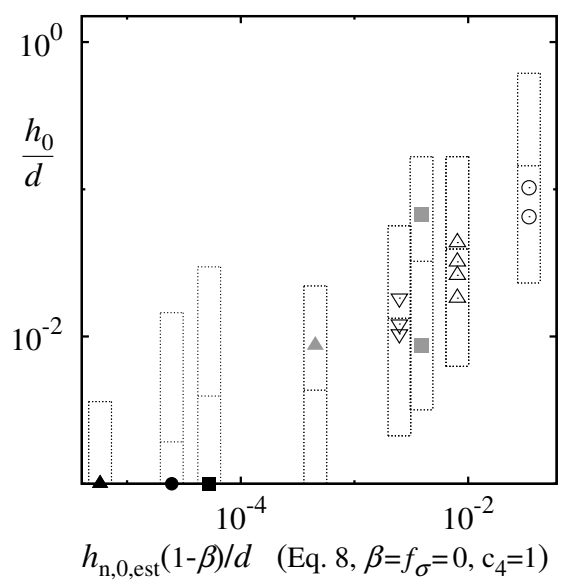

(b)

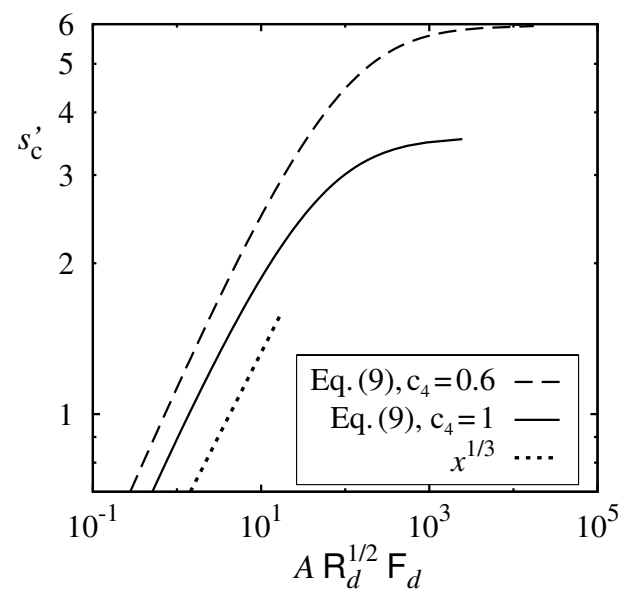

Figure 5 (a) Comparison of measured (symbols) and estimated (boxes) tip depths. (b) Predicted scaling behaviour of the critical submergence.

bers is $\mathrm{R}_{\mathrm{P}} / \mathrm{R}_{\mathrm{M}}=\alpha^{3 / 2}$. If it is further assumed that the velocity profiles outside and inside the vortex follow the same shape in the model and prototype so that $\left\{\left[d /\left(c_{4} s\right)\right]-c_{2}\right\}$ and $(1-\beta)$ are identical in the model and prototype, we obtain

$$
\frac{h_{\mathrm{P}}^{\prime}}{h_{\mathrm{M}}^{\prime}}=\frac{\mathrm{R}_{\mathrm{P}}}{\mathrm{R}_{\mathrm{M}}}=\alpha^{3 / 2}
$$

This indicates that the vortex depression $h_{\mathrm{P}}^{\prime}$ produced in the prototype would be significantly greater in relative terms than that $h_{\mathrm{M}}^{\prime}$ produced in the laboratory model if the scaling ratio $\alpha$ is large scaling ratios of 20 are common and can reach as high as 200 in some cases (Hecker, 1981). This predicted result is due to the fact that the vicosity of the fluid is identical in both model and prototype while circulation increases with intake size and is thus larger in the prototype.

This result is contradicted by observations of decreasing sensitivity to Reynolds number at large R values (Daggett and Keulegan, 1974; Jain et al., 1978; Anwar, 1983; Chang and Prosser, 1987), as well as the high success rate of laboratory-scale modelling for predicting prototype vortex activity in the past (Hecker, 1981; Montilla et al., 2004). The discrepancy suggests that additional processes or changes in flow structure must come into play at larger scales. To begin with, the laboratory and prototype intakes could have different axial profiles $V_{z}(z)$, producing $\beta_{\mathrm{M}} \neq \beta_{\mathrm{P}}$, and/or slightly different flow structures outside the vortex, whereas they are assumed to be identical. If turbulent diffusion enhances the effective viscosity controlling $r_{o}$ at larger scales and/or higher flow rates as suggested by Einstein and Li (1951), Anwar (1969) and Odgaard (1986), this would reduce the value of $h_{\sigma, 0}$ at larger scales. To properly assess this possibility, it would be necessary to make simultaneous measurements of the $V_{z}(z)$ and $V_{\theta}(r)$ profiles such as those made in Suerich-Gulick (2013), but at larger scales and $R$ values. 
Increased turbulence in the surrounding flow might also modify scaling behaviour at higher $R$ values by preventing vortices from forming or intensifying, as observed by Padmanabhan and Hecker (1984) and Tastan and Yıldırım (2010). Work on the interaction of external turbulence with the trailing vortices produced in the wake of airplane wings indicates that its impact depends on the length- and time-scale characteristics of both the background turbulence and of the central vortex of interest (Zeman, 1995; Jacquin and Pantano, 2002; Beninati and Marshall, 2005).

\section{Conclusion}

The free surface profile calculations reveal that both the shape and the scale of the free surface profile determine how surface tension will modify the shape and total depth of the depression. The results suggest that the magnitude of surface tension effects in a laboratory-scale vortex can be fairly easily estimated using the proposed correction factor. In comparison, scale effects linked to viscosity appear to be much more difficult to explain and predict. By incorporating the non-linear velocity profile of the flow surrounding the vortex, the proposed analytical model successfully reproduces the decreasing sensitivity to Froude number observed experimentally by earlier authors at deep submergences. However the model in its current form fails to reproduce the independence from Reynolds number observed by previous authors at large Reynolds values, suggesting that additional processes must intervene or that the flow structure may change at larger scales.

Potential changes in the flow structure at larger scales, as well as perturbations and enhanced diffusion caused by turbulence will have to be examined more closely to better understand the observed scaling behaviour. The proposed vortex model linking vortex characteristics to intake geometry and approach flow is a useful tool to evaluate how different processes interact to control vortex characteristics at both the laboratory scale and at larger scales. $* * *$ One sentence removed here. $* * *$

\section{Acknowledgements}

We would like to acknowledge the advice and contributions of David Morissette, Pierre Tadeo, Tristan Aubel, Maryse Page, Anne-Marie Giroux and Sébastien Houde. F.S.-G. was supported by the National Science and Engineering Research Council, the Fonds Québécois pour les Sciences et les Technologies, and Hydro-Québec's research institute.

\section{Notation}

$a \quad=$ axial gradient of axial velocity $\left[\mathrm{s}^{-1}\right]$ 


$$
\begin{aligned}
& \mathrm{c}_{1-6}=\text { non-dimensional model coefficients [-] } \\
& d \quad=\text { intake pipe inner diameter }[\mathrm{m}] \\
& f_{\sigma} \quad=\text { surface tension correction function [-] } \\
& \mathrm{F} \quad=\text { intake Froude number [-] } \\
& g \quad=\text { gravitational acceleration }\left[\mathrm{ms}^{-2}\right] \\
& h_{0} \quad=\text { free surface depression tip depth [m] } \\
& h^{\prime} \quad=\text { non-dimensional depression tip depth [-] } \\
& h_{r} \quad=\text { local derivative of } h \text { w.r.t. } r \text { [-] } \\
& h_{r} \quad=\text { local second derivative of } h \text { w.r.t. } r\left[\mathrm{~m}^{-1}\right] \\
& k=\text { distance between the piers [m] } \\
& K=\text { viscous correction factor [-] } \\
& l_{p} \quad=\text { pier length }[\mathrm{m}] \\
& \ell \quad=\text { intake characteristic length }[\mathrm{m}] \\
& l_{\sigma} \quad=\text { air-water interface characteristic length [m] } \\
& N_{v} \quad=\text { non-dimensional vicosity parameter [-] } \\
& N_{\Gamma *} \quad=\text { non-dimensional circulation [-] } \\
& \mathrm{Q} \quad=\text { intake flow rate }\left[\mathrm{m}^{3} \mathrm{~s}^{-1}\right] \\
& \mathrm{R}=\text { Reynolds number [-] } \\
& r=\text { vortex radial coordinate }[\mathrm{m}] \\
& r_{o} \quad=\text { vortex characteristic radius [m] } \\
& s \quad=\text { intake submergence }[\mathrm{m}] \\
& s_{\mathrm{c}}^{\prime} \quad=\text { non-dimendional critical submergence [-] } \\
& U_{i} \quad=\text { mean intake velocity }\left[\mathrm{ms}^{-1}\right] \\
& \mathrm{W}=\text { intake Weber number [-] } \\
& \alpha \quad=\text { model-to-prototype scaling factor [-] } \\
& \beta=V_{z} \text { linearization ratio [-] } \\
& \triangle h \quad=\text { tip depth difference due to surface tension [m] } \\
& \chi \quad=\text { eddy diffusivity scaling coefficient [-] } \\
& \delta \quad=\text { relative surface tension effect [-] } \\
& \Gamma_{\infty}=\text { vortex bulk circulation }\left[\mathrm{m}^{2} \mathrm{~s}^{-1}\right]
\end{aligned}
$$




$$
\begin{array}{ll}
\kappa & =\text { local mean free surface curvature }\left[\mathrm{m}^{-1}\right] \\
v & =\text { water molecular viscosity }\left[\mathrm{m}^{2} \mathrm{~s}^{-1}\right] \\
v_{\mathrm{eff}} & =\text { effective viscosity }\left[\mathrm{m}^{2} \mathrm{~s}^{-1}\right] \\
v_{\mathrm{T}} & =\text { eddy diffusivity }\left[\mathrm{m}^{2} \mathrm{~s}^{-1}\right] \\
\pi & =\text { trigonometric constant } \\
\theta & =\text { vortex azimuthal coordinate }[\mathrm{rad}] \\
\rho & =\text { water density }\left[\mathrm{kg} \mathrm{m}^{-3}\right] \\
\zeta & =\text { nominal depression slope }[-]
\end{array}
$$

\section{Subscripts}

$0 \quad=$ value at $(r=0)$

$\mathrm{M} \quad=$ laboratory model value

$\mathrm{P} \quad=$ prototype value

$d \quad=$ value based on intake diameter

est = estimated value

$\mathrm{n} \quad=$ without surface tension effect

$s \quad=$ value based on intake submergence

$\sigma \quad=$ with surface tension effect

\section{References}

Andersen, A., Bohr, T., Stenum, B., Rasmussen, J.J., Lautrup, B. (2006). The bathtub vortex in a rotating container. J. Fluid Mech. 556, 121-146.

Anwar, H.O. (1969). Turbulent flow in a vortex. J. Hydraulic Res. 7(1), 1-29.

Anwar, H.O. (1983). The non-dimensional parameters of free-surface vortices measured for horizontal and vertically inverted intakes. Houille Blanche 1, 11-25.

Anwar, H.O., Amphlett, M.B. (1980). Vortices at vertically inverted intake. J. Hydraulic Res. 18(2), $123-134$.

Beninati, M.L., Marshall, J.S. (2005). External turbulence interaction with a columnar vortex. $J$. Fluid Mech. 540, 221-245.

Bradshaw, P. (1973). Effects of streamline curvature on turbulent flow. AGARDograph No. 169 .

Burgers, J.M. (1948). A mathematical model illustrating the theory of turbulence. Adv. in Appl. Mech. 1, 171-199.

Chang, E., Prosser, M.J. (1987). Basic results of theoretical and experimental work. In J. Knauss, ed., Swirling flow problems at intakes, 39-55, A.A. Balkema, Rotterdam. 
Daggett, L.L., Keulegan, G.H. (1974). Similitude in free-surface vortex formations. J. Hydraulic Div. 100(HY11), 1565-1581.

Einstein, H.A., Li, H. (1951). Steady vortex flow in a real fluid. Proc. Heat Trans. Fluid Mech. Inst. $33-43$.

Gulliver, J.S. (1988). Discussion of Free-surface air core vortex. J. Hydraulic Div. ASCE 114(4), $447-449$.

Hecker, G. (1981). Model-prototype comparison of free surface vortices. J. Hydraulic Div. ASCE 107(HY10), 1243-1259.

Hite, J.E., Mih, W.C. (1994). Velocity of air-core vortices at hydraulic intakes. J. Hydraulic Eng. 120(3), 284-297.

Ito, K., Sakai, T., Eguchi, Y., Monji, H., Ohshima, H., Uchibori, A., Xu, Y. (2010). Improvement of gas entrainment prediction method - introduction of surface tension effect. J. Nucl. Sci. Technol. 47(9), 771-778.

Jacquin, L., Pantano, C. (2002). On the persistence of trailing vortices. J. Fluid Mech. 471, 159-168.

Jain, A.K., Raju, K.G.R., Garde, R.J. (1978). Vortex formation at vertical pipe intakes. J. Hydraulic Div. ASCE 104(HY10), 1429-1445.

Jiming, M., Yuanbo, L., Jitang, H. (2000). Minimum submergence before double-entrance pressure intakes. J. Hydraulic Eng. 126(8), 628-631.

Knauss, J. (1987). Prediction of critical submergence. In Swirling flow problems at intakes, 57-76, J. Knauss, ed. Balkema, Rotterdam NL.

Laplace, P.S. (1807). 2e supplément à la théorie de l'action capillaire. In Traité de mécanique céleste, Vol. 4, livre X, Courcier, Paris.

Möller, G., Meyer, A., Detert, M., Boes, R. (2012). Lufteintragsrate durch Einlaufwirbel - modellfamilie nach froude. In Proc. Int. Conf. Internationales Wasserbausymposium, Graz, AT.

Montilla, G., Marcano, A., Castro, C. (2004). Air entrainment at Guri Dam intake operating at low heads. In Proc. Int. Conf. Hydraulics of Dams and River Structures, Tehran, Iran, 53-60, F. Yazdandoost, J. Attari, eds., Balkema, Rotterdam, NL.

Odgaard, A. (1986). Free-surface air core vortex. J. Hydraulic Eng. 112(7), 610-620.

Padmanabhan, M., Hecker, G.E. (1984). Scale effects in pump sump models. J. Hydraulic Eng. 110(HY11), 1540-1556. 
Quick, M. (1962). Scale relationships between geometrically similar free spiral vortices, Pt. 2. Civ. Eng. Public Works Rev. 57, 1319-1320.

Rao, S., Diwanji, V., Srivastava, Y. (1997). Discussion of Critical submergence for intakes in open channel flow. J. Hydraulic Eng. 123(6), 588-589.

Spalart, P.R. (1998). Airplane trailing vortices. Annu. Rev. Fluid Mech. 30, 107-138.

Squire, H.B. (1965). The growth of a vortex in turbulent flow. Aeronaut. Quart. 16(Pt.3), 302-306.

Stepanyants, Y., Yeoh, G. (2008a). Burgers-Rott vortices with surface tension. Z. Angew. Math. Phys. 59, 1057-1068.

Stepanyants, Y., Yeoh, G. (2008b). Stationary bathtub vortices and a critical regime of liquid discharge. J. Fluid Mech. 604, 77-98.

Suerich-Gulick, F. (2013). Axial stretching, viscosity, surface tension and turbulence in free surface vortices at low-head hydropower intakes. Ph.D. thesis, McGill University.

Suerich-Gulick, F., Gaskin, S.J., Parkinson, E., Villeneuve, M. (2014). The characteristics of free surface vortices at low-head hydropower intakes. J. Hydraulic Eng. (in press) .

Tastan, K., Yıldırım, N. (2010). Effects of dimensionless parameters on air-entraining vortices. $J$. Hydraulic Res. 48(1), 57-64.

Yıldırım, N., Jain, S. (1981). Surface tension effect on profile of a free vortex. J. Hydraulic Div. ASCE 107(HY1), 132-136.

Yıldırım, N., Kocabaş, F. (1995). Critical submergence for intakes in open channel flow. $J . H y$ draulic Eng. 121(12), 900-905.

Zeman, O. (1995). The persistence of trailing vortices: a modeling study. Phys. Fluids 7(1), 135143. 